\title{
COST EFFECTIVE WEAPON SYSTEM DEVELOPMENT THROUGH INTEGRATED MODELING AND HARDWARE TESTING
}

\author{
Larry H. Johnson \\ Charles M. Crocker, Jr. \\ U.S. Army Test and Evaluation Command \\ Redstone Technical Test Center \\ Redstone Arsenal, Alabama 35898-8052, U.S.A.
}

\begin{abstract}
Weapon system development has historically relied heavily on both hardware testing and computer modeling and simulation. Testing has generally been a test-fix-test approach, beginning at the component and subsystem level with increasing complexity up to and including system level field testing. Modeling and simulation have been utilized extensively to investigate and solve complex or high risk technical problems such as missile guidance techniques, etc. These tools have served well in the past; however, today's environment of increasing weapon system complexity with higher cost and shrinking Defense resources require change. These tools have been integrated somewhat in the past on a case by case basis, but not in a consistent manner. This paper suggests cost effective change by tailoring and integrating these reliable tools in a systematic way that allows development of reliable and quality systems at lower cost and in less time.
\end{abstract}

\section{INTRODUCTION}

This paper presents an approach for integrating modeling and simulation with test and evaluation to improve both processes and develop weapon systems in a more cost effective manner. Modelers and testers need to combine their efforts early in the weapon system development program in such a way that:

a. modeling and simulation still meets the system/hardware designer's needs

b. modeling and simulation are utilized to determine what testing is needed, what data needs to be collected, to analyze test results, and to limit or minimize the amount of hardware testing needed for system development

c. every test conducted serves the dual purpose of evaluating hardware performance and validating the model/simulation.

\section{APPROACH}

From the modeling perspective, computer models need to be structured or subdivided into stand alone modules that correlate with hardware components and subsystems. These modules could then be combined to represent the major subsystems and, ultimately, the total weapon system as depicted in Figure 1. These modules need to accommodate capabilities for data input and output that replicate the hardware (with applicable embedded software) that they are intended to represent.

Each module and combination of modules could be validated through a structured hardware component/ subsystem test program. Once the models are validated, hardware and software modules could be utilized interchangeably for hardware-in-the-loop testing as depicted in Figure 2. This represents a modified approach to modeling and simulation.

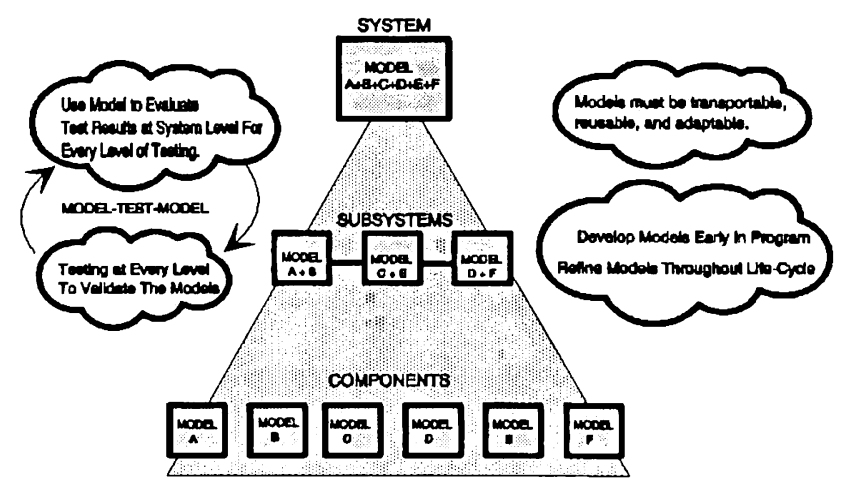

Figure 1: Bottom-Up/Modular Modeling Concept

From the testing perspective, the requirement to answer a myriad of questions concerning system performance in a host of operational scenarios will not change. However, a well structured and integrated test/hardware-in-the-loop program can provide the needed data for any number of scenario combinations with a minimum number of expensive hardware tests. Structured modeling will facilitate testing at the component/subsystem level and transforming results for 
system level evaluation. Every test conducted (component, subsystem and system) should be conducted for the dual purpose evaluating hardware performance and validating computer models. The resulting process could be described as test-model-test as opposed to testfix-test. This represents a modified approach to hardware testing.

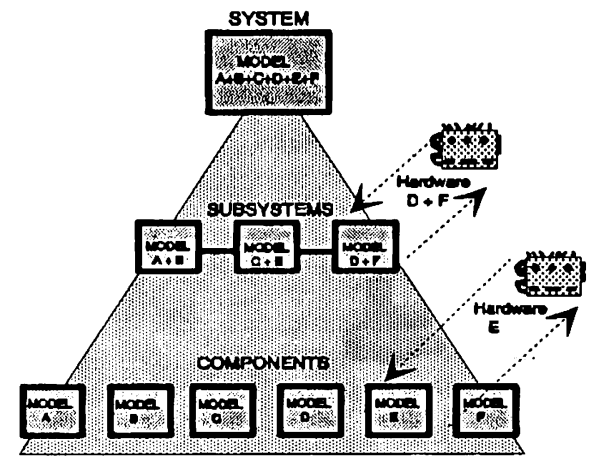

Figure 2: Interchangeable Hardware and Software

Component/subsystem development and qualification testing will be conducted as it has been conducted in the past; however, the results will be compared with pre-test modeling predictions to validate the models and combined with the system model to transform results to the system level for evaluation. This process is depicted in Figure 3.

This integrated process will provide "real world" environmental effects on hardware performance for incorporation in the models. Testing will be repeatable and can be conducted under conditions not practical in field tests because of physical limitations, safety, etc.

From the modeling perspective, the proposed methodology will provide detailed information about the weapon system at a fidelity and uniformity not generally available in the past. Engineers will be able to study and analyze the performance of each component and interactions within/between subsystems to determine the "weak links" and where to improve the design to develop a reliable and performing system. The key is to validate each level of the model (component through system) by hardware testing.

\section{LONG TERM BENEFITS}

The integrated modeling and hardware testing will support the total weapon system life cycle starting with concept studies and continue until the system is taken out of the inventory; however, the models must be developed and validated through testing during the research and development program phase.

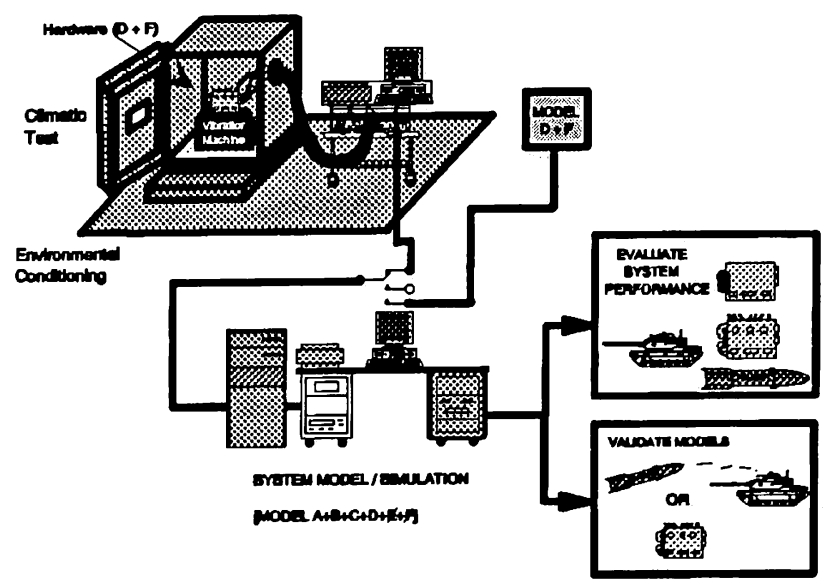

Figure 3: Integrated Modeling and Testing

By modeling and testing in this manner, libraries and databases can be developed such that new concepts can be modeled and studied before actually building hardware prototypes.

Modeling and simulation will become a "tool" much like conventional test instrumentation for the tester and evaluator.

\section{CHALLENGES}

This approach to developing weapon systems requires a change in "mind-set" on behalf of most participants. Modelers, designers, testers and evaluators will be required to stay out of "turf" battles and work together as a team in a cooperative manner. Each will be required to change the way they have done things in the past to optimize the overall process. Confidence and support from project managers and acquisition decision makers will be required to give the process opportunity to prove its worth.

Modeling architecture and protocols must be standardized to a degree that requirements can be specified in contracts, and resulting models can be "run" by modelers, testers and evaluators across a broad base of computing systems.

\section{CONCLUSIONS}

The concept proposed herein is not new, but it is an application whose time has come. The need for more efficiencies in the development process and today's low cost/high speed computers provide the necessary initiatives and opportunities to develop this process. The advantages and opportunities are limited only by our paradigms of the past. 


\section{AUTHOR BIOGRAPHIES}

LARRY H. JOHNSON is Director of the U.S. Army Redstone Technical Test Center at Redstone Arsenal, Alabama. The Center is part of the U.S. Army Test and Evaluation Command and is responsible for providing test and evaluation personnel and facility support to the missile system development and acquisition community at Redstone Arsenal. He received a B.S. degree in petroleum engineering from Texas Technological College in 1959 and a M.S.E. degree from the University of Alabama in Huntsville in 1969. As Director of the Center, his interests are focused on optimizing the test and evaluation process and providing reliable and performing hardware to the soldier at an economical cost. As result of these interests, he has been instrumental in the initiation and integration of modeling and simulation with the hardware test and evaluation process.

CHARLES M. CROCKER, JR, is Chief of the Electronic Component Test Branch of the Redstone Technical Test Center, USATECOM, at Redstone Arsenal, Alabama. This Branch is responsible for test and evaluation of the full spectrum of electronic and microwave/millimeter wave components, assemblies, sub-systems and systems utilized in USAMICOM weapon systems. Mr. Crocker received a B.S. degree in electrical engineering from the University of Alabama in 1972. He is currently assigned as the RTTC Lead Engineer for Modeling and Simulation (M\&S) and is serving as the Center point of contact for M\&S activities. His primary responsibility in this area involves coordination of the Center's early planning and development efforts in the merger of the Center's existing hardware test capabilities with advanced computer simulation technologies. 